\title{
Nutrition and Food in the Reproduction of Cattle
}

\author{
Alejandro Córdova-Izquierdo* and Blanca Estela Rodriguez Denis \\ Department of Agricultural and Animal Production, Universidad Autónoma Metropolitana Unidad Xochimilco, Mexico
}

*Corresponding author: Alejandro Córdova Izquierdo, Department of Agricultural and Animal Production, Autonomous Metropolitan University Unit Xochimilco, Mexico.

To Cite This Article: Alejandro Córdova Izquierdo. Nutrition and Food in the Reproduction of Cattle. Am J Biomed Sci \& Res. 2019 - 6(1). AJBSR. MS.ID.001000. DOI: 10.34297/AJBSR.2019.06.001000.

Received: 眥 October 30th, 2019; Published: 眥 November 06, 2019

\section{Opinion}

At the beginning of the 1980s, a series of very profound changes were initiated in the milk cow nutrition approaches, because of the highest levels of production per cow that were reached by the productive systems of the northern hemisphere. Nutrition is defined as the series of processes through which an organism acquires and assimilates food to promote its growth and replace worn or damaged tissues. The nutrients are fundamental for the animals to carry out their different productive functions.

When we consider the aspects that touch the field of nutrition of ruminants, we understand the importance of this group of animals of zootechnical interest, which can process plant components that are not consumed by other mammals, the structural carbohydrates (fiber). Ruminant comes from the word "rumen", which is the largest of the compartments in the stomach of four compartments of a bovine, sheep or goat. This structure is where microbial fermentation takes place.

The ruminants, through evolutionary processes, developed life relationships with microorganisms which enabled them to use fiber as food, that is, they developed in some way their "food factory". They eat the forage to be transformed by the rumen's microbiota into substances that are the source of energy for the animal and for the microbial synthesis, the microbial cells are an excellent source of proteins for the animal. However, the processes that make the ruminal microbiota are, in a certain way, inefficient. Grass degradation produces volatile fatty acids, microbial protein and gases. Within these gases, some are environmental pollutants such as $\mathrm{CO} 2$, methane and nitrous oxide.

Millions of bacteria, protozoa, and fungi live in the rumen and degrade parts of the plant rich in energy, making them digestible to the animal host. After the forage has been digested in the rumen and degraded to smaller parts, it can pass through the reticulum and omasum, which function as colanders that trap large pieces of material preventing them from reaching the abomasum, or "true stomach", where digestion continues.

The nutritional concern for ruminants focuses on energy (ie, carbohydrates), protein, minerals, vitamins, and water. The energy (carbohydrates) is responsible for the functions of growth and maintenance of the animal, and the generation of heat. The protein makes the tissue grow and performs other vital functions. Other nutrients and minerals such as vitamin A and E, calcium, phosphorus, and selenium can be fed to "free choice" as a mineral supplement.

Dairy cows of high productive potential (9000-12000 / liters / lactation) currently represent a real challenge for nutrition. For many years, there has been evidence of the impact of nutrition on the reproductive behavior of the bovine female Florez et al., 2007. The main factor that affects reproduction is the undernourishment due to the scarcity and quality of the food. Subsequent research has shown that nutritional effects are exerted through complex interrelations between various aspects such as: content and use of body reserves, distribution of nutrients between different systems and organs and prioritization of the use of nutrients for various functions in addition to reproduction.

\section{Foundations of Animal Nutrition}

Animal nutrition is the part of animal husbandry that studies the use of different foods - or, more specifically, the immediate principles that constitute them - to satisfy the needs of animals useful to man. This is defined as the series of processes through which an organism acquires and assimilates food to promote its growth and replace worn or damaged tissues. The nutrients are fundamental for the animals to carry out their different productive functions.

Nutrients absorbed from the digestive tract include volatile fatty acids, glucose, minerals, and vitamins. These are used in the 
synthesis of many different compounds found in meat, milk and wool, and to replace nutrients used to support living processes including reproduction.

For the purposes of calculating nutrition, they are usually divided into two groups:

Of support: they are those that allow the animals to cover their minimum needs to continue living, although without any type of production.

Of production: they are those that, once covered the necessities of support, allow the animals to produce something useful for the man: meat, milk, young, work, etc.

The total food needs are calculated by adding the support and production needs. The nutritional needs of animals with respect to reproduction are critical, to achieve an adequate level of maintenance. Undernutrition during growth produces delays in sexual maturity. If there are nutrient deficiencies before the mating season, they can render animals sterile, produce low fertility or fail to maintain and establish pregnancy.

On the other hand, nutritional requirements should be taken as basic and fundamental within the diet, considering the intake, which is defined as the ingestion of nutrients by the animal, and is regulated by factors that in turn interrelate as:

Palatability, it is the flavor and texture of the food. Ruminants seek sweetness in their food, probably because the sweet taste is an indicator of soluble carbohydrates, the most critical element of the diet for the animal after water. Ruminants avoid bitter flavors, which are usually associated with toxic secondary chemicals.

The foraging behavior. describes how the animal performs the foraging process. According to Fred Provenza, a pastoralist researcher at UTA State University, the study of foraging behavior involves

understanding:

a. Habits of food and habitat preferences, and

b. The effects of nutrients and toxins of preference.
The bite size and the bite rate also influence the intake. The denser a pasture, the more forage the animal can take with each bite. This exemplifies the fact that the relationship between grazing management, animal behavior, and nutrient intake is not a simple relationship. It is a complex and constantly changing relationship that follows changes in seasons, forage quality, and amount of forage.

Chemical factors include nutrients, but also secondary chemicals that are usually associated with the defense of the plant. It usually refers to secondary chemicals as toxic substances, but the toxicity depends on the degree, or dosage. All plants contain secondary chemicals to some degree, but animals have evolved an innate sense of what is good to eat.

Animals limit the number of plants they consume that contain secondary chemicals through a feedback mechanism, which results in satiety, or the feeling that they have eaten enough. According to Webster, satiety is the "quality or state of being fed or gratified up to or beyond the capacity point, or the repulsion or disgust caused by overindulgence or excess. When ruminants consume enough of a certain toxic substance, a feedback mechanism induces a switch to an alternative source of nutrients. This is the reason why cattle, sheep and goats graze more (have a higher intake) in a diverse pasture. Variety stimulates their appetite and provides alternative sources when they have reached the limit of their preferred source of food.

Quantity, density and availability of forage directly influence the intake of forage, and intake is directly related to the density of the meadow. Ruminants can only take a limited number of bites per minute as they graze, and cattle only graze for 8 hours a day. Therefore, it is important to make sure that each bite taken by the animal is as large as possible. A bovine graze surrounding the forage with its tongue and then tearing it upwards; sheep and goats use their lips and teeth to select highly nutritious parts of the plant. Large bites of forage are therefore insured by maintaining high density of pastures. 